\title{
Super-resolution imaging goes fast and deep
}

\author{
Sam Duwé \& Peter Dedecker*
}

Lab for Nanobiology, KULeuven, Leuven, Belgium

\section{Advances in image scanning microscopy (ISM) move super-resolution imaging deeperinto tissues with faster visualization and finer details.}

Fluorescence microscopy is the imaging technique of choice for many researchers, and even more so with the advent of super-resolution imaging. While these techniques can offer a dramatically increased spatial resolution, they do come with various limitations, such as slower measurements and higher light doses. Over the past few years, work by several research groups has resulted in the development of image scanning microscopy (ISM), a method that increases the spatial resolution of confocal instruments by up to a factor of two, without fundamentally penalizing their performance or applicability. Now, two developments reported by the laboratories of Shroff ${ }^{1}$ and Enderlein ${ }^{2}$ dramatically enhance ISM performance in two-photon excitation (2PE) microscopy, resulting in deeper and faster imaging, while also expanding its use to second harmonic generation (SHG) microscopy

It has long been known that adding an infinitely small pinhole to an optical microscope can improve its spatial resolution up to two-fold. Unfortunately, this al so decimates the detectable signal, so the pinholes in actual confocal instruments are much larger, sacrificing most of the resolution improvement. ISM enables the use of the system as if it has a much smaller pinhole, achieving the full resolution improvement, without compromising the sensitivity or imaging speed. ${ }^{3}$ 
The key to ISM is to replace the single pinhole and detector with a grid of detectors, each of which acts like a tiny pinhole. While one such detector detects considerably less signal, there is a net gain in detected signal because the grid covers the entire emission spot. Most of these detector pixels are located away from the center of focus (the optical axis), and collect fluorescence from a sample region that is likewise shifted from the optical axis (Figure 1A). This is where a key aspect of ISM comes in: even though the detector in Figure $1 \mathrm{~A}$ can in principle record emission for the full region highlighted by the green curve, this fluorescence can only arise from those parts of the sample illuminated by the excitation light (purple curve). As a result, the recorded fluorescence arises only from an area centered halfway between the green and purple curves (blue curve in Figure 1B). In unmodified imaging this emission is thus assigned to an incorrect position. ISM corrects this by shifting the light detected in every pixel to its actual origin (figure $1 \mathrm{C}$ ), and then creating an image by adding these corrected emission spots for all positions on the sample. Because the corrected light distribution is narrower, the final image has a higher spatial resolution. Importantly, ISM does not introduce any distortions and does not depend on the sample or fluorophores, since it simply compensates for the fact that the optics treat excitation and emission independently, despite their being intrinsically linked.

The key requirement for ISM is a grid detector that can sample the emission spot, such as a sCMOS camera. The initial implementation of ISM imaged every sample position sequentially on the camera and computationally reconstructed the ISMimage, leading to impractically long acquisition times. ${ }^{4}$ Recent adaptations of ISM recognized that reducing the emission spot two-fold in size is equivalent to sweeping the sample emission across a camera that is optically four times larger than the excitation scan area, while preserving the spot size. ${ }^{5,6}$ This 'all optical' ISMis much faster, computation-free and can be realized using e.g. two optical scanners, one to scan the beam across the sample and another to increase the sweep area on the camera. Compared to its nearest analog in terms of performance, structured illumination microscopy (SIM), all-optical ISM sidesteps the tricky computational reconstruction of SIM, does not have issues with out-of-focus fluorescence, and is 
easier to realize. However, the need for scanner synchronization orlarge-area scanners meant that only galvanometer scanners could be used, which are much slower and less robust than the combination of resonant/galvanometer scanners typically used in confocal instruments, leading to a strongly reduced imaging speed.

Deep-tissue imaging using 2PE is increasingly important, but has proven difficult to combine with super-resolution microscopy. ISM can be directly applied due to its similarity to confocal imaging. ${ }^{7}$ However, this sparked limited interest due the reduced imaging speed, impeding e.g. fast neuronal imaging, and because the additional information exploited in ISM deteriorates rapidly in the presence of optical distortions, such as when imaging deeper.

The work by Zheng et al. applies adaptive optics (AO) to enable much deeper imaging with their 2PE implementation of ISM, which they call instant structured illumination microscopy (iSIM). They first de-scan the emission over a wavefront sensor to characterize the distortions, after which a deformable mirror is used to correct the excitation and emission light during the actual image acquisition. The net result is the impressive acquisition of high quality ISM images up to $250 \mu \mathrm{m}$ in the sample, more than doubling the accessible imaging depth.

The work by Gregor et al. describes an extremely elegant 2PE-ISM implementation which strongly reduces the instrumental complexity while also matching the imaging speed of conventional $2 \mathrm{PE}$ microscopy ( $\sim 30 \mathrm{~Hz}$ with a resonant scanner). Their core insight is that, instead of using two scanners, the light can be 'looped' over the same scanner before imaging onto a camera, simplifying the instrument while retaining the full benefits of ISM. In addition, the authors describe different post-processing approaches to maximize the available information, and cover new ground in demonstrating the use of ISMin second harmonic generation (SHG) imaging.

Both developments dramatically expand the applicability of ISM and break new ground in the combination of 2PE and super-resolution imaging, enabling highly detailed studies in e.g. brain, heart and tumor tissues, where optical penetration can be an issue. That said, both systems have 
limitations. The AO implementation uses the dual-scanner approach, which reduces its imaging rate to about $1 \mathrm{~Hz}$, requires expensive hardware, and required additional fluorophores administered to the sample for wavefront sensing. Unfortunately, there is no readily-apparent way to combine this strategy with the instrument by Gregor et al. In turn, the main limitation of the latter instrument is that it is only suitable for non-linear imaging (e.g. 2PE or SHG), since the continuous motion of the emission beam precludes the introduction of a physical pinhole needed for classical confocal sectioning.

Nonetheless both instruments are highly powerful, and the design of the single-scanner instrument is such that it can be home-built by groups with moderate optical expertise. In the long run, however, it is likely that the camera-scanning based approach will be replaced by systems using dedicated detector arrays, similar to what is currently being used in the proprietary Zeiss AiryScan system. In addition to eliminating the need for multiple scanners or scanner passes, such a system would also enable the combination of ISM with e.g. fluorescence lifetime imaging. With these new developments we fully expect that, except for cases specifically demanding non-descanned detection, ISM setups will supplant classical confocal microscopes.

Competing Financial Interests statement:

The authors declare no competing financial interests 


\section{References:}

1. Zheng, W. et al. Nat. Methods 14, 869-872 (2017).

2. Gregor, I. et al. Nat. Methods (2017).

3. Sheppard, C. Optik (Stuttg). 80, 53-54 (1988).

4. Müller, C. B. \& Enderlein, J. Phys. Rev. Lett. 104, 198101 (2010).

5. De Luca, G. M. R. et al. Biomed. Opt. Express 4, 2644 (2013).

6. Roth, S., Sheppard, C. J., Wicker, K. \& Heintzmann, R. Opt. Nanoscopy 2, 5 (2013).

7. Winter, P. W. et al. Optica 1, 181 (2014).

8. Schulz, O. et al. Proc. Natl. Acad. Sci. 110, 21000-21005 (2013).

Figures and Captions:

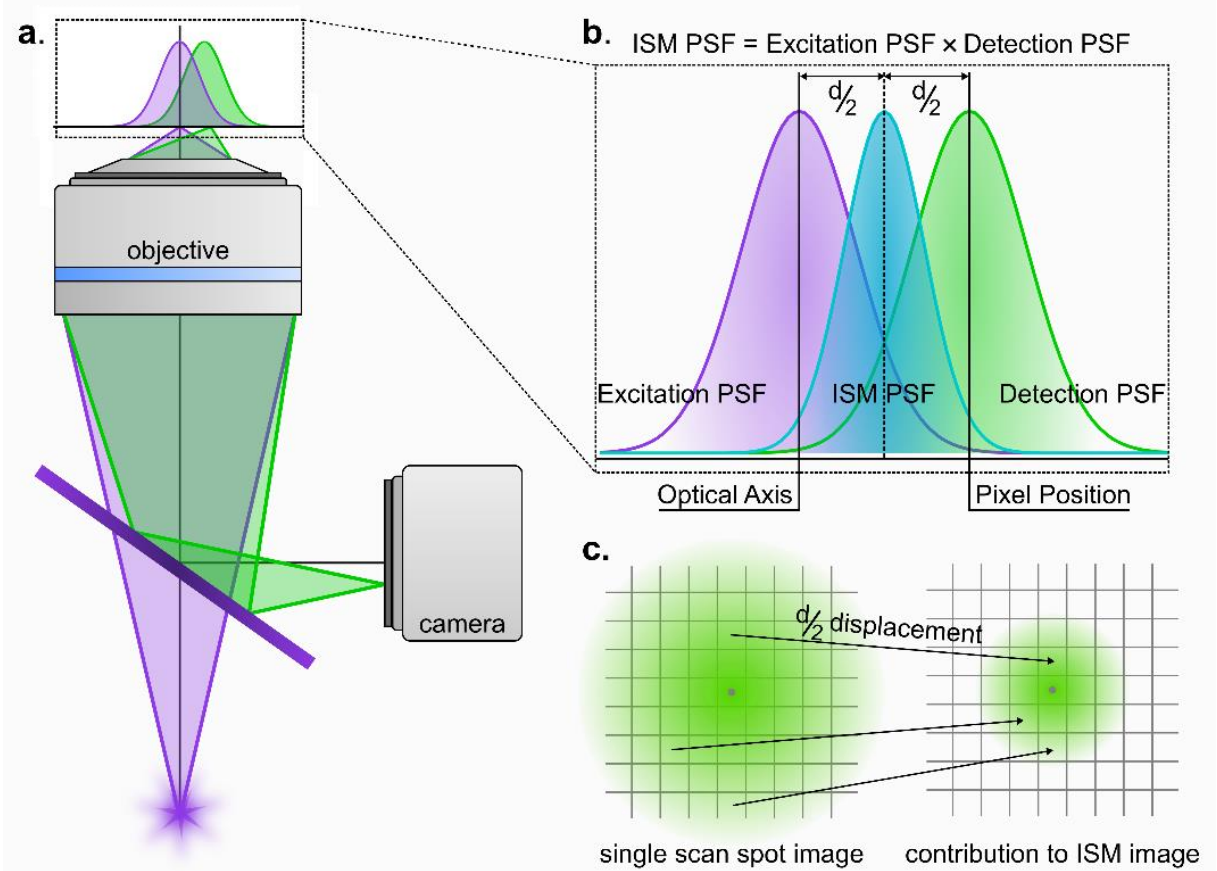

Figure 1: (a) Fluorescence detected by detector pixels not on the optical axis originate from sample areas that are similarly not on the optical axis. (b) The detected fluorescence originates from an area that is half the distance (d/2) closer to the optical axis than perceived by the detector pixel. (c) ISM corrects for this by displacing the detected fluorescence towards the optical axis by d/2. ISM: image scanning microscopy, PSF: point spread function. Panel A and B based on Ref. ${ }^{8}$. 